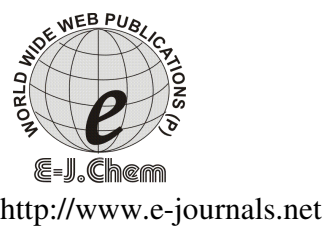

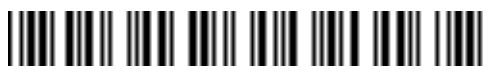

ISSN: 0973-4945; CODEN ECJHAO

E-Journal of Chemistry

Vol. 4, No. 2, pp 166-172, April 2007

\title{
Synthesis and Antibiotic Activity of Mebendazole Derivatives of Pharmacological Interest
}

\author{
KAVITA RATHORE, USHA AMETA, SWATI OJHA, \\ RANJANA SHARMA and G. L. TALESARA* \\ Synthetic Organic Chemistry Laboratory, \\ Deptt. of Chemistry, M. L. Sukhadia, University, Udaipur - 313001 India. \\ E-mail: usha_ameta@yahoo.com,gtalesara@yahoo.com
}

Received 20 October 2006; Accepted 15 November 2006

\begin{abstract}
Mebendazole is a well known anti-helimintic and belongs to the benzimidazole group of medicines. In order to achieve better medicinal results, i.e. enhanced activity and low toxicity, structural modifications are made in the existing drugs. Some 5-benzoyl-N-[1-(alkoxyphthalimido) benzimidazol-2-yl] carbamic acid methyl ester (3a-c) and 5-benzoyl-N-[1-(2,3-bis oxyphthalimido/oxysuccinimido propyl benzimidazol-2-yl) carbamic acid methyl ester (7a-b) have been synthesized from two different routes. Structures of the compounds have been established on the basis of elemental analysis and spectral studies. All the synthesized compounds (3a-c) and (7a-b) were assayed in vitro for antimicrobial activity against mebendazole (itself) and standard [ciprofloxacin (antibacterial) and fluconazole (antifungal)].
\end{abstract}

Keywords: Antimicrobial activity, Mebendazole, Alkoxyphthalimide, Benzimidazole, Anthelimintics.

\section{Introduction}

Mebendazole (me-BEN-da-zole) belongs to the family of medicines called anthelmintics, is especially useful against whipworm infection. It kills mostly nematodes and some cestodes. It is used principally to treat infections due to roundworms (ascariases), hookworms (uncinarians), pinworms (enterobiasis), whipworms and also used in the treatment of capillarasis ${ }^{1}$. Further low doses of mebendazole, selectively and reversibly block glucose uptake by nematodes, whereas even the high dose of it does not affect glucose levels in mammals ${ }^{2}$. Mebendazoles (MBZ) are widely used in veterinary medicines. Mebendazole was found effective in decreasing the concentration of a strain of cytochromes P450 (CYP) in primary culture of swine (Sus scrofaf. domestica) hepatocytes ${ }^{3}$. Pre-and post intervention 
chemotherapy with mebendazole offers the advantage of reducing the risk of cystic echinococcosis of the liver recurrence and intraperitoneal seeding of infection ${ }^{4}$. Mebendazole has been tested as antigiradial agents, which acts by especially inhibiting tubulin polymerisation caused by Giardia lambia, a parasite survives in the intestinal tracts of human and other mammals as microtubule assembly ${ }^{5}$. Basically, mebendazole is a benzimidazole derivative. Benzimidazole derivatives have also attracted considerable attention due to their potent diversified bioactivities such as inhibitors of influenza virus ${ }^{6,7}$, RNA viruses ${ }^{8}, \mathrm{HIV}^{9}$, breast cancer cells(MCF7) ${ }^{10}$ and Helicobacter pylori ${ }^{11}$. Thesa also posses analgesic ${ }^{12,13}$, antiinflammatory ${ }^{13,14}$, central nervous system depressant ${ }^{15}$ properties.

Various aminooxy compounds also exhibit diverse biological properties. 1-Aminoxy-3aminopropane is a potent competitive inhibitor of mammalian ${ }^{16,17}$ and bacterial ${ }^{18}$ ornithine $^{2}$ decarboxylase, the inhibition being due to a direct and very tight, but reversible, binding of drug to the enzyme ${ }^{19}$. Further more, the methyl-N-phthalimidoxy-2-methylacrylate (E49) against a different cell lines, had a potential anticancer activity ${ }^{20}$. Spirosuccinimides compounds offered varying degrees of anticonvulsant effects in electrically and chemically induced seizures ${ }^{21}$. Many alkoxyphthalimide derivatives possess anticonvulsant ${ }^{22}$, anticancer ${ }^{23}$, antimicrobial and antimalarial ${ }^{24,25}$ activities.

In view of the immense biological activities of these heterocycles, it was thought to construct a system, which may combine these heterocyclic rings in molecular framework containing both moieties to study the resultant medicinal effect. Hence, it was planned to synthesize titled molecules and to evaluate their antimicrobial activity.

\section{Experimental}

\section{Materials}

The reagent grade chemical were purchased from commercial sources and purified by either distillation or recrystallisation before use.

\section{Instruments}

Melting points were determined by open capillary method and are uncorrected. IR spectra $(\mathrm{KBr})$ were recorded on perkin- Elmer 1800 FTIR and Jasco FTIR - 410 spectrophotometer. ${ }^{1} \mathrm{H}$ NMR spectra $\left(\mathrm{CDCl}_{3} / \mathrm{CF}_{3} \mathrm{COOH}\right)$ were recorded on a Bruker DRX $300\left(300 \mathrm{MH}_{\mathrm{z}} \mathrm{FT}\right.$ NMR) and varion spectrometer ( $270 \mathrm{MH}$ ) using TMS as internal standard and mass spectra were determined on a Jeol D- 300 (EI) spectrometer.

\section{Sodium salt of 5-Benzoyl(1H)benzimidazol-2-yl-carbamic acid methyl ester (1)}

To a solution of mebendazole (2.45 gm, 0.01 mole) in DMF taken in a stoppered conical flask, $\mathrm{Na}$ metal $(23 \mathrm{gm})$ or sodium ethoxide $(6.2 \mathrm{gm})$ or $\mathrm{NaOH}(24 \mathrm{gm})$ was added portionwise, till effervescence ceased. Solid (1) was separated on cooling which was used as such.

\section{5-Benzoyl-N-[1-(alkoxy phthalimido)benzimidazol-2-yl]carbamic acid methyl ester (3)}

Sodium salt of mebendazole (1) $(3.17 \mathrm{gm}, 0.01 \mathrm{~mole})$ was suspended in DMF, phthalimidoxyethylbromide (2.69gm, 0.01 mole) in DMF was added with constant stirring on a powerful magnetic stirrer. The mixture was further stirred for next 14 hrs till the solution becomes light yellow. Crystals of sodium bromide were filtered and the filtrate was added to 200gm crushed ice, solid (3) separated, was recrystallised by absolute alcohol.

Compounds $\mathbf{3 b}$ and $\mathbf{3} \mathbf{c}$ were also prepared by similar method with minor changes in reflux time etc. 
3a Compounds : IR(KBr)cm ${ }^{-1}: 3351$ (N-H str.), 3099 (C-Hstr.,Ar-H), 2921(C-Hstr., $\mathrm{CH}_{3}$ ), 2828 (C-H str., $\mathrm{CH}_{2}$ ), 1721 (C=Ostr.), 1688 (CONH), 1622 ((C=Nstr.), 1033 (C-Nstr.), 955 (N-O str.), 755 (C-H def.,1,2 disubstituted benz.), 699 (C-H bend., monosub. benz) ${ }^{1} \mathrm{HNMR}\left(\mathrm{CDCl}_{3}\right)(\mathrm{ppm}) \delta: 7-6.3(\mathrm{~s}, 1 \mathrm{H}, \mathrm{NH}), 7.81-7.73(\mathrm{~m}, 12 \mathrm{H}, \mathrm{Ar}-\mathrm{H}), 3.51\left(\mathrm{t}, 2 \mathrm{H}, \mathrm{CH}_{2} \mathrm{O}\right)$, 2.99((t, $\left.2 \mathrm{H}, \mathrm{CH}_{2} \mathrm{~N}\right), 2.11\left(\mathrm{~s}, 3 \mathrm{H}, \mathrm{OCH}_{3}\right): \mathrm{MS}(\mathrm{m} / \mathrm{z}) 468\left[\mathrm{C}_{26} \mathrm{H}_{20} 0_{5} \mathrm{~N}_{4}\right]^{+\cdot}$ or $[\mathrm{M}]^{+\cdot} 278,173,190$, $132,115,105,104,77$.

3b Compounds: IR (KBr)cm ${ }^{-1}: 3299$ (N-H str.), 3061 (C-H str., Ar-H), $1715(\mathrm{C}=\mathrm{O}), 1681$ (CONH), 2881 (C-H str., $\mathrm{CH}_{2}$ ), 2931 (C-H str., $\left.\mathrm{CH}_{3}\right), 1629$ (C=N str.),1121 (C-N str.), 981 ( $\mathrm{N}-\mathrm{O}$ str.); ${ }^{1} \mathrm{HNMR}\left(\mathrm{CDCl}_{3}\right)(\mathrm{ppm}) \delta$ : 7.81-7.61 (m, 12H, Ar-H], 7.54 (s, IH, NH), 3.43 (t, $2 \mathrm{H}, \mathrm{CH}_{2}-\mathrm{N}$ ), 2.01 (quint, $2 \mathrm{H}, \mathrm{CH}_{2} \mathbf{C H}_{2} \mathrm{CH}_{2}$ ); $\mathrm{MS}(\mathrm{m} / \mathrm{z}) 482\left[\mathrm{C}_{27} \mathrm{H}_{22} \mathrm{O}_{5} \mathrm{NH}_{4}\right]^{+\cdot}$ or $[\mathrm{M}]^{+\cdot}$ 278, 204, 173,162, 132, 115, 105, 77, 58, 43, 28.

3c Compound: IR(KBr)cm ${ }^{-1}$ : 3318 (N-H str.), 3028 (C-Hstr.,Ar-H), 2968 (C-Hstr., $\mathrm{CH}_{3}$ ), 2832 (C-H str., $\left.\mathrm{CH}_{2}\right), 1711(\mathrm{C}=\mathrm{O}), 1698(\mathrm{CONH}), 1633(\mathrm{C}=\mathrm{N}), 933$ (N-Ostr.), 1131 $(\mathrm{C}=$ Nstr. $) ;{ }^{1} \mathrm{HNMR}\left(\mathrm{CDCl}_{3}\right)(\mathrm{ppm}) \delta$ : 7.74-7.63 (m, 12H, Ar-H), $7.11(\mathrm{~s}, 1 \mathrm{H}, \mathrm{NH}), 3.44$ (t, $2 \mathrm{H}, \mathrm{CH}_{2} \mathrm{O}$ ), 2.75 (t, 2H, $\mathrm{CH}_{2}-\mathrm{N}$ ), 2.17 (multiplet, $4 \mathrm{H}, \mathrm{CH}_{2} \mathrm{CH}_{2}$ ), 2.03 (s, 3H, $\mathrm{OCH}_{3}$ ) MS $(\mathrm{m} / \mathrm{z}) 496\left[\mathrm{C}_{28} \mathrm{H}_{24} \mathrm{O}_{5} \mathrm{~N}_{4}\right]^{+*}$ or $[\mathrm{M}]^{+*} 278,218,173,162,132,115,105,77,76,58,28$.

\section{5-Benzoyl-N-[1-(allyl)benzimidazol-2-yl]carbamic acid methyl ester (5)}

To the stirred solution of sodium salt of mebendazole ((1), $6.34 \mathrm{~g}, 0.02 \mathrm{~mole})$ in DMF, allyl bromide ( $2.4 \mathrm{~mL}, 0.02$ mole) was added drop wise with a dropping funnel and the stirring was continued for next $9 \mathrm{~h}$ till the solution became light yellow. It was filtered to remove $\mathrm{NaBr}$ and poured into 100 gm crushed ice. The clumpy solid separated was triturated with $10 \mathrm{~mL}$ of methanol. Crystalline solid (5) was filtered, dried and recrystallised by ethanol.

IR (KBr)cm ${ }^{-1}$ : 3378 (N-H str.), 3048 (C-H str., Ar-H), 2958 (C-H str., $\mathrm{CH}_{3}$ ), 2825 (C-H str., $\left.\mathrm{CH}_{2}\right), 1711(\mathrm{C}=\mathrm{O}), 1668(\mathrm{CONH}), 1614(\mathrm{C}=\mathrm{N}), 1017$ (C-Nstr.), $826(\mathrm{C}-\mathrm{H}$ bend., trisubs. benzene ring); ${ }^{1} \mathrm{HNMR}\left(\mathrm{CDCl}_{3}\right) \mathrm{ppm} \delta$ : 7.71-7.43 (m, 8H, Ar-H), 7.08 (s, 1H, CONH), 4.42 (d, $2 \mathrm{H}, \mathrm{CH}_{2}$ ), 4.01 (quint, $1 \mathrm{H}, \mathrm{CH}=\mathrm{CH}_{2}$ ), 3.19 (d, $\left.2 \mathrm{H}, \mathrm{CH}_{2}-\mathrm{CH}=\right), 2.13\left(\mathrm{~s}, 3 \mathrm{H}, \mathrm{OCH}_{3}\right.$ ), MS $(\mathrm{m} / \mathrm{z}) 319\left[\mathrm{C}_{19} \mathrm{H}_{17} \mathrm{O}_{2} \mathrm{~N}_{3}\right]^{+}$or $[\mathrm{M}]^{+.}, 278,173,115,105,77,75,58,43,28,15$.

\section{5-Benzoyl-N-[1-(2,3-dibromopropyl)benzimidazol-2-yl]carbamic acid methyl ester (6)}

To a stirred solution $(\mathbf{5}, 3.35 \mathrm{gm}, 0.01 \mathrm{~mole})$ in chloroform, (1.66gm, $0.01 \mathrm{~mole})$ of bromine in $10 \mathrm{~mL}$. chloroform was added drop wise during $1 \mathrm{~h}$. The light yellow colored solid was obtained which turned into white colored solid after the crystallization with ethanol.

IR(KBr) cm ${ }^{-1}: 3304$ (N-H str.), 3111 (C-H str., Ar-H), 2933 (C-H str., $\mathrm{CH}_{3}$ ), 2822 $\left(\mathrm{C}-\mathrm{H}\right.$ str. $\left.\mathrm{CH}_{2}\right), 1708(\mathrm{C}=\mathrm{O}), 1688(\mathrm{C}=\mathrm{O}, \mathrm{CONH}), 691$ (C-Br str.) 706 (C-H def., 1,2-disub.benz.); ${ }^{1} \mathrm{HNMR}\left(\mathrm{CDCl}_{3}\right)(\mathrm{ppm}) \delta$ : 7.9-7.8 (m, 8H, Ar-H), $5.8(\mathrm{~s}, 1 \mathrm{H}, \mathrm{NH}), 2.8(\mathrm{~d}$, $\left.2 \mathrm{H}, \mathrm{CH}_{2}\right), 2.6\left(\mathrm{~d}, 2 \mathrm{H}, \mathrm{CH}_{2}\right) ; \mathrm{MS}(\mathrm{m} / \mathrm{z}) 479\left[\mathrm{C}_{19} \mathrm{H}_{17} \mathrm{O}_{2} \mathrm{~N}_{3} \mathrm{Br}_{2}\right]^{+}$or $[\mathrm{M}]^{+}, 481,278,275,173$, $115,105,77,58,43,28,15$.

\section{5-Benzoyl-N-[1-(2,3-bis phthalimidoxy propyl)benzimidazol-2-yl]carbamic acid methyl ester (7a)}

To a stirred solution of $(\mathbf{6}, 6.5 \mathrm{gm}, 0.01 \mathrm{~mole})$ and triethylamine $(0.02 \mathrm{~mole})$ in DMF, a concentrated solution of N-hydroxyphthalimide (1.63 gm. 0.01 mole) in DMF was added drop wise at room temperature. The reaction mixture was stirred for four hours and kept over right at room temperature. Mixture was filtered and the filtrate was poured into crushed ice. Solid obtained was dried and recrystallised from ethanol. 
$\operatorname{IR}(\mathrm{KBr}) \mathrm{cm}^{-1}: 3311$ (N-H str), 2931 (C-Hstr. $\left.\mathrm{CH}_{3}\right), 2811\left(\mathrm{C}-\mathrm{H}\right.$ str., $\left.\mathrm{CH}_{2}\right), 1715(\mathrm{C}=\mathrm{O}), 1681$ $\left(\mathrm{C}=\mathrm{O}\right.$ str.,CONH), $1611(\mathrm{C}=\mathrm{N}), 1018(\mathrm{C}-\mathrm{N}), 925(\mathrm{~N}-\mathrm{O}),{ }^{1} \mathrm{HNMR}\left(\mathrm{DMSO}_{6}\right)(\mathrm{ppm}) \delta: 7.71$ (m, 16H, Ar-H), 7.32 (s, $1 \mathrm{H}, \mathrm{CONH}), 3.91\left(\mathrm{~d}, 2 \mathrm{H}, \mathrm{CH}_{2}\right), 3.31$ (quint, $1 \mathrm{H}, \mathrm{CH}_{2}-\mathbf{C H}-\mathrm{CH}_{2}$ ), $2.13\left(\mathrm{~d}, 2 \mathrm{H}, \mathrm{CH}_{2}\right), 2.07\left(\mathrm{~s}, 3 \mathrm{H}, \mathrm{OCH}_{3}\right) ; \mathrm{MS}(\mathrm{m} / \mathrm{z}) 659\left(\mathrm{C}_{35} \mathrm{H}_{25} \mathrm{O}_{8} \mathrm{~N}_{5}\right)^{+^{+}}$or $[\mathrm{M}]^{+\cdot} 278,283,190$, $162,132,115,105$.

\section{5-Benzoyl-N-[1-(2,3-bis succinimidoxy propyl)benzimidazol-2-yl]carbamic acid methyl ester (7b)}

Dibromo compound $(\mathbf{6}, 4.925 \mathrm{gm}, 0.01$ mole) dissolved in DMF, a solution of two moles of $\mathrm{N}$-hydroxysuccinimide (1.15 gm, $0.01 \mathrm{~mole}$ ) in DMF, was added to it slowly with constant stirring and triethylamine ( $2.02 \mathrm{gm}, 0.02 \mathrm{~mole})$ was added. The contents were stirred for $8 \mathrm{~h}$ and kept for $16 \mathrm{~h}$ at room temperature. Solid tri-ethylammonium bromide was filtered and the filtrate was poured into $(150 \mathrm{gm})$ crushed ice. Solid obtained after filtration was dried and recrystallised from ethanol.

$\operatorname{IR}(\mathrm{KBr}) \mathrm{cm}^{-1}$ : 3299 (N-H str.), 3011 (C-H str., Ar-H), 1714 (C=O), 1681 (C=Ostr.,CONH), $1611(\mathrm{C}=\mathrm{N}), 1019(\mathrm{C}-\mathrm{N}), 899\left(\mathrm{~N}-\mathrm{O}\right.$ str.); ${ }^{1} \mathrm{HNMR}\left(\mathrm{DMSO}_{6}\right) \delta(\mathrm{ppm}): 7.64(\mathrm{~m}, 8 \mathrm{H}, \mathrm{Ar}-\mathrm{H})$, $7.41(\mathrm{~s}, 1 \mathrm{H}, \mathrm{NH}) 5.1\left(\mathrm{dd}, 2 \mathrm{H}, \mathrm{CH}_{2}\right) 3.84\left(\mathrm{~d}, 2 \mathrm{H}, \mathrm{CH}_{2}\right), 3.03$ (qunit, $\left.1 \mathrm{H}, \mathrm{CH}_{2}-\mathbf{C H}-\mathrm{CH}_{2}\right) 2.11$ (d, $\left.2 \mathrm{H}, \mathrm{CH}_{2}\right), 2.00\left(\mathrm{~s}, 3 \mathrm{H}, \mathrm{OCH}_{3}\right) . \mathrm{MS}(\mathrm{m} / \mathrm{z}): 563[\mathrm{M}]^{+}, 449,173,114,105,98$.

\section{Results and Discussion}

Mebendazole has active hydrogen at position 1, which can be replaced by a suitable functionality using strong bases. Although a number of bases have tried, sodium hydride was found better. Various mebendazole derivatives were prepared as shown in the scheme 1. Mebendazole in DMF solution was treated with sodium hydride very slowly and portionwise. Sodium salt (1), thus formed was treated with a solution of $\omega$-bromoalkoxyphthalimide in DMF. N-H peak at $3351 \mathrm{~cm}^{-1}$ was disappeared in the product. In another route the sodium salt was reacted with allyl bromide. Allyl mebendazole so formed was treated with bromine to give dibromo compound (6). Treatment of dibromo compound with $\mathrm{N}$-hydroxyphthalimide and $\mathrm{N}$ - hydroxysuccinimide gave the bis product $(\mathbf{7 a - b}),>\mathrm{C}=\mathrm{C}<$ at $1648 \mathrm{~cm}^{-1}$ was disappeared in dibromo compound and characteristic CO-N-CO peak appeared at $1740 \mathrm{~cm}^{-1}$.

Structural assignments of compounds 3a-c, 5, 6 \& 7a-b are based on spectral and analytical studies. For compound 3a, ethoxyphthalimide derivative (the gross formula $\mathrm{C}_{26} \mathrm{H}_{20} \mathrm{~N}_{4} \mathrm{O}_{6}$ ) was confirmed by the mass spectrum, which exhibited a molecular ion at $\mathrm{m} / \mathrm{z}$ 484. The ${ }^{1} \mathrm{HNMR}$ spectrum of 3a-c displayed signals at $\delta 3.43-3.51\left(\mathrm{t}, 2 \mathrm{H}, \mathrm{CH}_{2}-\mathrm{O}\right), 2.72-$ $2.99\left(\mathrm{t}, 2 \mathrm{H}, \mathrm{CH}_{2}-\mathrm{N}\right) \& 2.12-2.03\left(\mathrm{~s}, 3 \mathrm{H}, \mathrm{OCH}_{3}\right)$. Moreover, the structure of compound 5 has been assigned on the basis of elemental analysis supporting the gross formula $\mathrm{C}_{19} \mathrm{H}_{17} \mathrm{~N}_{3} \mathrm{O}_{3}$. This was also confirmed by the mass spectrum which gave a correct molecular ion peak at $\mathrm{m} / \mathrm{z}$ 335. ${ }^{1} \mathrm{H}$ NMR as well as IR spectra also establish the structure of compound $\mathbf{5}$. Structural proof of final compound $\mathbf{7 a - b}$ or phthalimido and succinimido derivatives rests on the mass spectrum, which clearly indicates that both bromine atoms were completely replaced by phthalimido and succinimido group as $[\mathrm{M}+4]^{+\cdot}[\mathrm{M}+2]^{+\cdot}$ Peaks appeared in compound 6 were disappeared and molecular ion peak at $659[\mathrm{M}]^{+\cdot}$ and $563[\mathrm{M}]^{+\cdot}$ reveals the formation of 7a-b. Correct elemental analysis, ${ }^{1} \mathrm{H}$ NMR and IR spectrum further supported

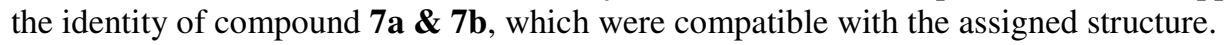


<smiles>COC(=O)Nc1nc2ccc(C(C)=O)cc2[nH]1</smiles>

(1)

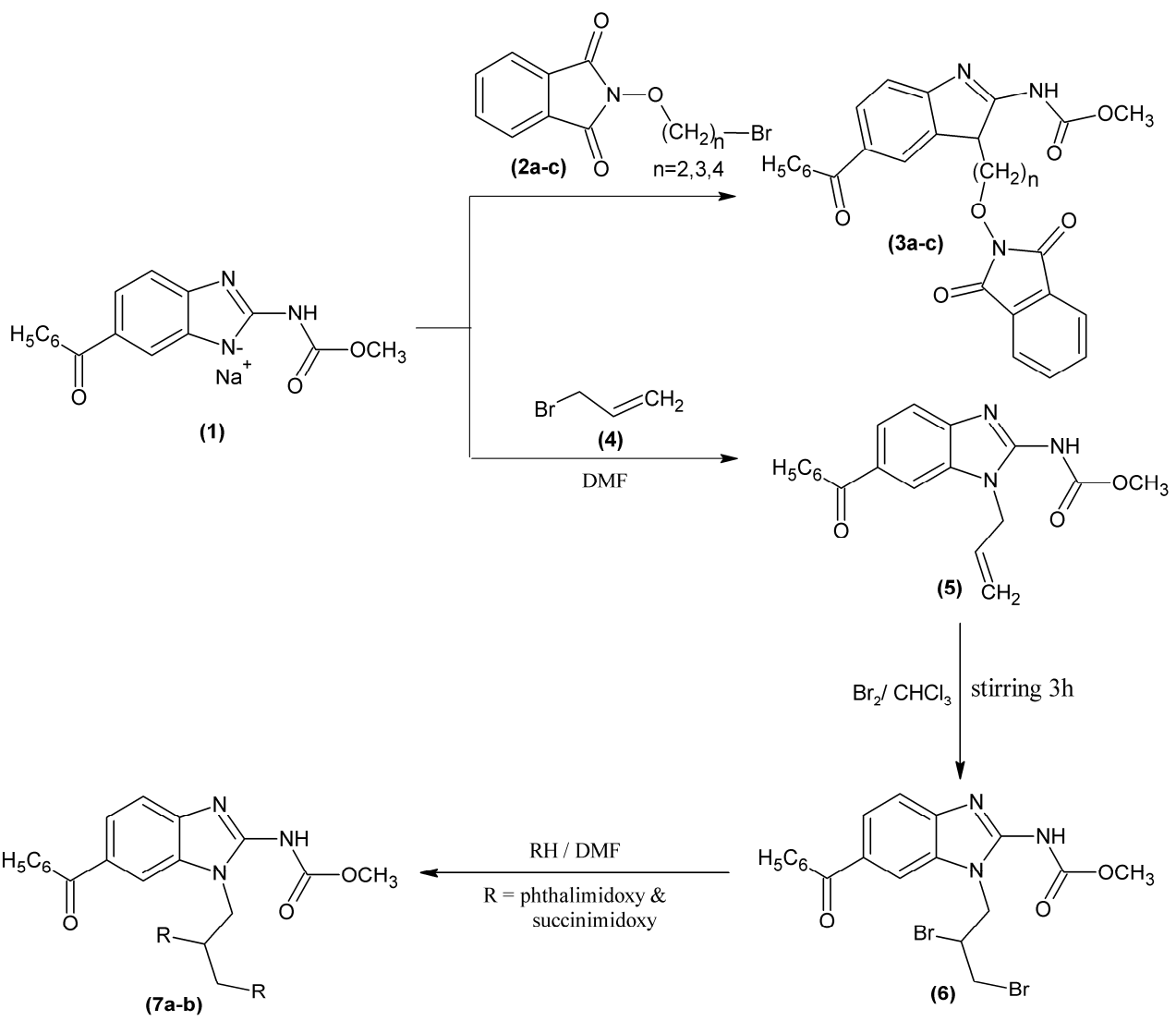

Scheme 1. Synthesis Routes to Mebendazole Derivatives (7a-b).

Table 1. Physical Data of Synthesized Compounds

\begin{tabular}{cccccccc}
\hline \multirow{2}{*}{ Comp. } & \multirow{2}{*}{ Mol. formula } & $\begin{array}{c}\text { Mol } \\
\text { Wt. }\end{array}$ & $\begin{array}{c}\text { M.P. } \\
\left({ }^{\circ} \mathrm{C}\right)\end{array}$ & $\mathrm{n}$ & $\begin{array}{c}\text { Yield } \\
\%\end{array}$ & \multicolumn{2}{c}{$\mathrm{N},(\%)$} \\
\hline 3a & $\mathrm{C}_{26} \mathrm{H}_{20} \mathrm{O}_{6} \mathrm{~N}_{4}$ & 484 & 295 & 2 & 55 & 11.57 & 10.52. \\
3b & $\mathrm{C}_{27} \mathrm{H}_{22} \mathrm{O}_{6} \mathrm{~N}_{4}$ & 498 & 298 & 3 & 58 & 11.24 & 11.01 \\
$\mathbf{3 c}$ & $\mathrm{C}_{28} \mathrm{H}_{24} \mathrm{O}_{6} \mathrm{~N}_{4}$ & 512 & 305 & 4 & 52 & 10.93 & 10.83 \\
$\mathbf{5}$ & $\mathrm{C}_{19} \mathrm{H}_{17} \mathrm{O}_{3} \mathrm{~N}_{3}$ & 335 & 185 & - & 44 & 12.53 & 11.98 \\
$\mathbf{6}$ & $\mathrm{C}_{19} \mathrm{H}_{17} \mathrm{O}_{3} \mathrm{~N}_{3} \mathrm{Br}$ & 495 & 190 & - & 47 & 8.48 & 8.60 \\
$\mathbf{7 a}$ & $\mathrm{C}_{35} \mathrm{H}_{25} \mathrm{O}_{9} \mathrm{~N}_{5}$ & 659 & 310 & - & 48 & 10.62 & 10.30 \\
$\mathbf{7 b}$ & $\mathrm{C}_{27} \mathrm{H}_{25} \mathrm{O}_{9} \mathrm{~N}_{5}$ & 563 & 280 & - & 42 & 12.43 & 11.90 \\
\hline
\end{tabular}




\section{Antimicrobial activity}

Antimicrobial activity was determined using disc diffusion method by measuring zone of inhibition area. All the synthesized ligands were screened for their antibacterial and antifungal activities against the E. coil, P. auregenosa, K., Pneumoniae, B. Subtilis, S. typhi and the fungi A. fungatus and $C$. albicans. The compounds were tested at $100 \mu \mathrm{g} / \mathrm{mL}$ concentration using $5 \mathrm{~mm}$ filter paper disc. Control experiment was carried out under similar condition by using ciprofloxacin for antimicrobial activity and fluconazole for antifungal activity as standard drugs.

\section{Comparison}

All the compounds were dissolved in DMF. The inhibition zone measured in mm showed that all compound 3a-c and 7a-b were found to be more active then MBZ against the above microbes. The results are displayed in Table 2.

The compound 3a and 7a exhibited good antifungal activity as compared to the standard fluconazole. Antimicrobial activity of $\mathbf{3 a - b}$ decreases when the length of alkyl chain $(n=2,3$, 4) is increased in alkoxyphthalimide moiety.

Table 2. Anti-microbial activity of synthesized compound 3a-c and 7a-b Zone of Inhibition in ( $\mathrm{mm})$ (activity index)

\begin{tabular}{|c|c|c|c|c|c|c|c|}
\hline \multirow[t]{2}{*}{ Comp. } & \multicolumn{5}{|c|}{$\begin{array}{c}\text { Antibacterial activity } \\
100(\mu \mathrm{g} / \mathrm{mL})\end{array}$} & \multicolumn{2}{|c|}{$\begin{array}{c}\text { Antifungal activity } \\
100(\mu \mathrm{g} / \mathrm{mL})\end{array}$} \\
\hline & E. coli & P aurengenosa & K pneumoniae & B subtilis & S. typhi & A.fumigatus & C.albicans \\
\hline \multirow[t]{3}{*}{ 3a } & 27 & 19 & 19 & 28 & 30 & 19 & 26 \\
\hline & $(1.28)^{*}$ & $(1.26)^{*}$ & $(1.18)^{*}$ & $(1.22)^{*}$ & $(1.25)^{*}$ & $(1.72)^{*}$ & $(1.85)^{*}$ \\
\hline & $(0.96)_{\mathrm{c} 1}$ & $(0.90)_{\mathrm{c} 1}$ & $(0.95)_{\mathrm{c} 1}$ & $(0.93)_{\mathrm{c} 1}$ & $(0.76)_{\mathrm{c} 1}$ & $(1.05)_{\mathrm{c} 2}$ & $(1.04)_{\mathrm{c} 2}$ \\
\hline \multirow[t]{3}{*}{$\mathbf{3 b}$} & 21 & 17 & 15 & 25 & 27 & 17 & 19 \\
\hline & $(1.00)^{*}$ & $(1.13)^{*}$ & $(0.93)^{*}$ & $(1.08)^{*}$ & $(1.12)^{*}$ & $(1.54)^{*}$ & $(1.35)^{*}$ \\
\hline & $(0.75)_{\mathrm{c} 1}$ & $(0.80)_{\mathrm{c} 1}$ & $(0.75)_{\mathrm{c} 1}$ & $(0.83)_{\mathrm{c} 1}$ & $(0.69)_{\mathrm{c} 1}$ & $(0.94)_{\mathrm{c} 2}$ & $(0.76)_{\mathrm{c} 2}$ \\
\hline \multirow[t]{3}{*}{$3 c$} & 25 & 17 & 18 & 27 & 28 & 18 & 25 \\
\hline & $(1.19)^{*}$ & $(1.13)^{*}$ & $(1.12)^{*}$ & $(1.17)^{*}$ & $(1.16)^{*}$ & $(1.63)^{*}$ & $(1.78)^{*}$ \\
\hline & $(0.89)_{\mathrm{c} 1}$ & $(0.80)_{\mathrm{c} 1}$ & $(0.90)_{\mathrm{c} 1}$ & $(0.90)_{\mathrm{c} 1}$ & $(0.71)_{\mathrm{c} 1}$ & $(1.00)_{\mathrm{c} 2}$ & $(1.10)_{\mathrm{c} 2}$ \\
\hline \multirow[t]{3}{*}{$7 a$} & 23 & 16 & 17 & 24 & 26 & 15 & 23 \\
\hline & $(1.09)^{*}$ & $(1.06)^{*}$ & $(1.06)^{*}$ & $(1.04)^{*}$ & $(1.08)^{*}$ & $(1.36)^{*}$ & $(1.64)^{*}$ \\
\hline & $(0.82)_{\mathrm{c} 1}$ & $(0.76)_{\mathrm{c} 1}$ & $(0.85)_{\mathrm{c} 1}$ & - & $(0.66)_{\mathrm{c} 1}$ & $(0.83)_{\mathrm{c} 2}$ & $(0.92)_{\mathrm{c} 2}$ \\
\hline \multirow[t]{3}{*}{$7 b$} & 22 & 15 & 14 & 23 & 25 & 12 & 21 \\
\hline & $(1.04)^{*}$ & $(1.00)^{*}$ & $(0.87)^{*}$ & $(1.00)^{*}$ & $(1.04)^{*}$ & $(1.09)^{*}$ & $(1.50)^{*}$ \\
\hline & $(0.78)_{\mathrm{cl}}$ & $(0.71)_{\mathrm{c} 1}$ & $(0.70)_{\mathrm{c} 1}$ & $(0.76)_{\mathrm{c} 1}$ & $(0.615)_{\mathrm{c} 1}$ & $(0.66)_{\mathrm{c} 2}$ & $(0.84)_{\mathrm{c} 2}$ \\
\hline \multirow[t]{2}{*}{$\mathrm{C}_{1} *$} & 21 & 15 & 16 & 23 & 24 & 11 & 14 \\
\hline & $(0.77)_{\mathrm{c} 1}$ & $(0.71)_{\mathrm{c} 1}$ & $(0.80)_{\mathrm{c} 1}$ & $(0.76)_{\mathrm{c} 1}$ & $(0.80)_{\mathrm{c} 1}$ & $(0.61)_{\mathrm{c} 2}$ & $(0.56)_{\mathrm{c} 1}$ \\
\hline $\mathrm{C}_{1}$ & 28 & 21 & 20 & 30 & 39 & - & - \\
\hline $\mathrm{C}_{2}$ & - & - & - & - & - & 18 & 25 \\
\hline
\end{tabular}

Activity index = Inhibition zone of the sample/ Inhibition zone of the standard for antibacterial activity $\mathrm{C}_{1}=$ ciprofloxacin and for antifungal activity $\mathrm{C}_{2}=$ fluconazole. The compound $\mathrm{C}_{1} *=$ mebendazole.

\section{Acknowledgements}

Authors are thankful to the Head, Department of Chemistry, M.L. Sukhadia University, Udaipur for providing Laboratory facilities and to the Director, 'RSIC, CDRI, Lucknow, India for providing spectral and analytical data. Authors are also thankful to Defense Laboratory, 
Jodhpur for providing IR spectra. Authors are grateful to microbial Research Laboratory, particularly Dr. Kanika Sharma Department of Botany, M.L. Sukhadia University for evaluating anti-microbial activity. One of the authors (Swati Ojha) is thankful to CSIR, New Delhi for providing financial assistance.

\section{References}

1. Ali M, Text Book of pharmaceutical organic chemistry. CBS publishers and Distributors, New Delhi, 1999, 78.

2. $\quad$ Bera T and Belsare D P, Ind J Chem 1992, 32B, 370.

3. Baliharova V, Velik J, Savilk M, Szotakovav B, Lamka J, Tahotona L and Skalov L, J of Verterinary Pharmacology and Therapeutics 2004, 27, 85.

4. $\quad$ Raymond A, Smego Jr and Peter S, Inter J of Infectious Diseases 2005, 9, 69.

5. Mariante R M, Vanani R G, Melo A L and Bechimol M, Experimenta Parasitology 2005, 110, 62 .

6. Hallinshead A C and Smith P L, J Pharmacol Exptl Therap 1958, 123, 54.

7. Pandey V K, Tusi Z, Tusi S, Joshi M N and Bajpai S K, Ind J Hetro Chem 2002, 111, 309.

8. $\quad$ Sidwell R W and Huffman J H, Appl Microbiol 1971, 22, 797.

9. Roth T, Morningstar M L, Boyer P L, Hughes S H, Buckheit R W Jr. and Michejda C J, J Med Chem 1997, 40(26), 4199 .

10. Ramla M M, Omar M A, El-Khamry A M and El-Diwani H I, Bioorganic \& Medicinal Chemistry, (In Press).

11. Kühler T C, Swanson M, Shcherbuchin V, Larsson H, Mellgård B, and Sjöström J E, J Med Chem 1998, 41(11), 1777.

12. Mullican M D, Wilson M W, Canner D T, Kastlan C R, Schrier D J and Dyer R D, J Med Chem 1999, 36, 1090.

13. Sondhi S M, Singh N, Kumar A, Lozach O and Meijer L, Bioorganic \& Medicinal Chemistry 2006, 14(11), 3758.

14. Patel V H, Fernandes P S and Vyas K A, Ind J Chem 1990, 29B, 135.

15. Menon M K, Clark W G and Aures D, Eur J pharmacol 1972, 19, 43.

16. Khomutov R M, Denisova G F, Khomutov A R, Belastatskaya K M, Shlosman R B and Artamonova, Bioorg Khim 1985, 11, 1574.

17. Khomutov R M, Hyvonen T, Karvonen E, Kauppinen L, Paolaness T, Paulin L, Eloranta T, Pajula R L, Adersson L C and Posa H, Biochem Biophy Res Commu 1985, 130, 596-602.

18. Paulin L, FEBS Lett. 1986, 202, 323.

19. Lapinjoki S, Eloranta T and Paso H, On the mechanism of ornithine decarboxylase inhibition by 3-aminooxypropylamine. In Biochemistry of Vitamin B6. Edited by Korpela T and Christen P: Birkhauser Verlag; 1987.

20. Edafiogho I, National Caner Institute Developmental Therapeutics Program personal Communication.

21. Tarver M L, Nichalson J M and Scott K R, J Pharm Science 1985, 74(7), 785.

22. Loscher W G, Eur J Pharmacol 1998, 342, 1.

23. Bihzad S, Al-zaid B, Edafiogho I W, Seventh Annual Health Science Poster Day, 2002, p 108.

24. Singh B, Mehta D, Baregama L K, Ind J Chem 2004, 43B, 1306.

25. Berger B J, Antimicrob Agent Chemo 2000, 44(9), 2540. 


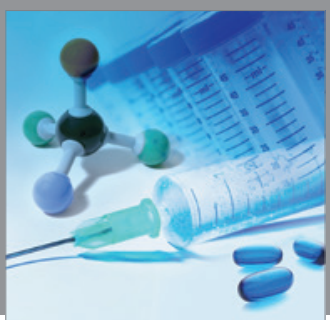

International Journal of

Medicinal Chemistry

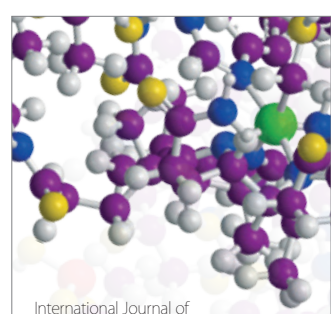

Carbohydrate Chemistry

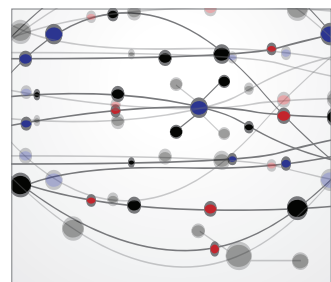

The Scientific World Journal
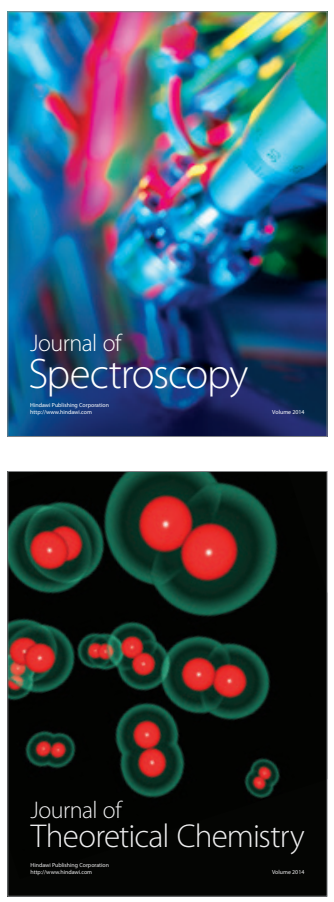
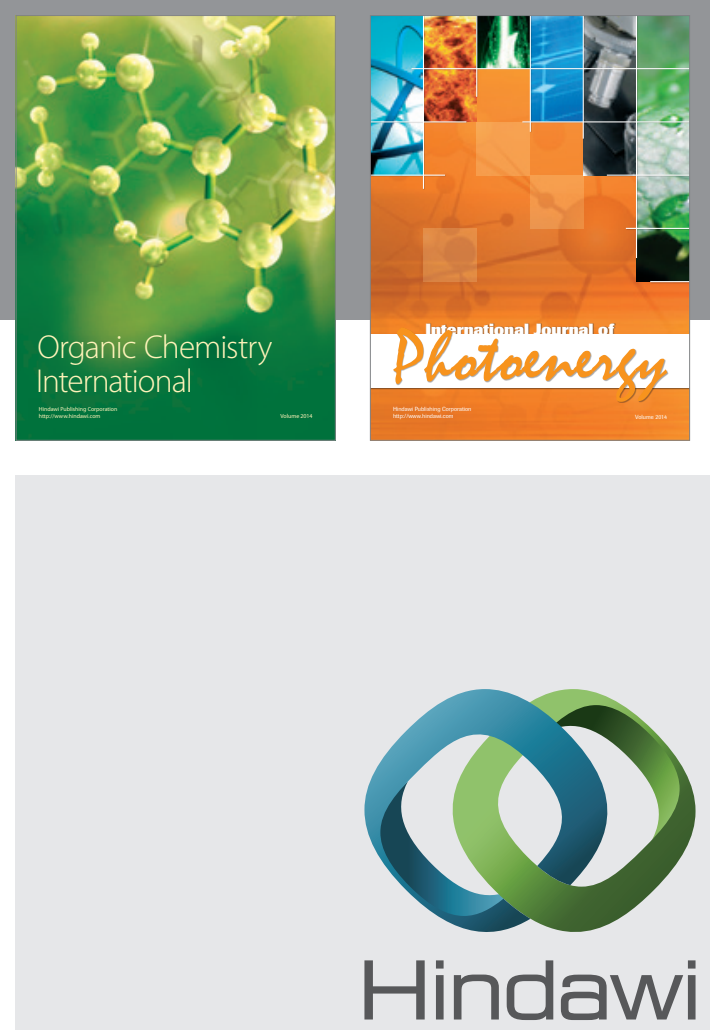

Submit your manuscripts at

http://www.hindawi.com
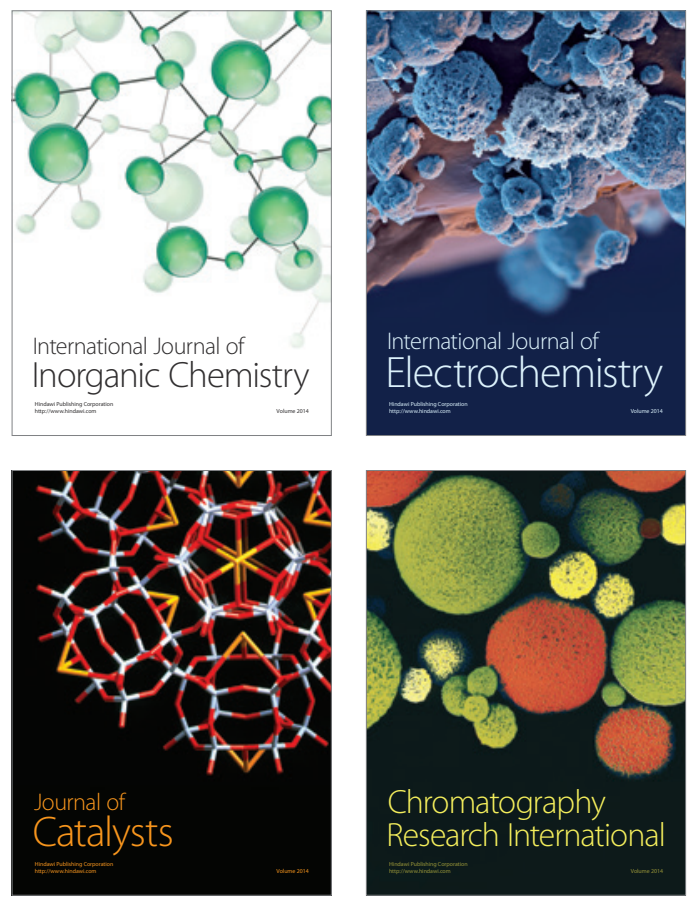
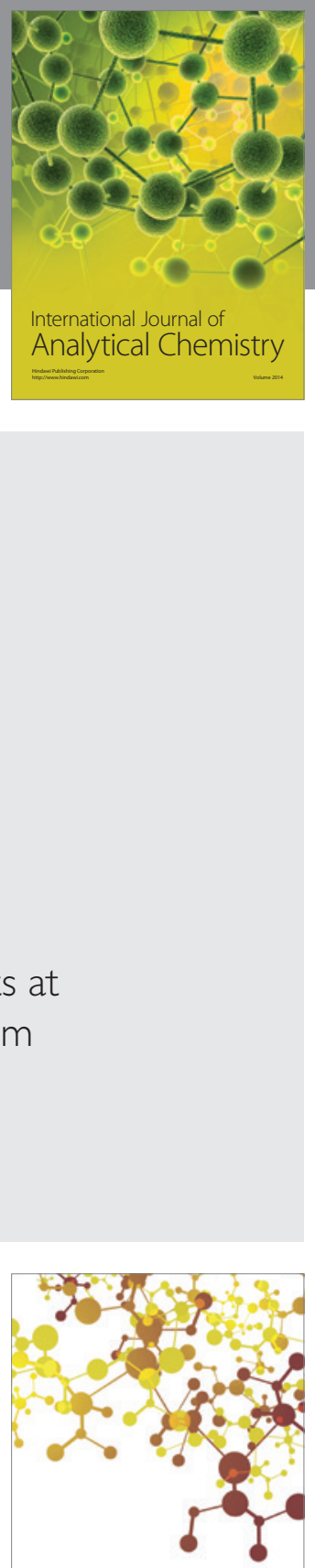

Journal of

Applied Chemistry
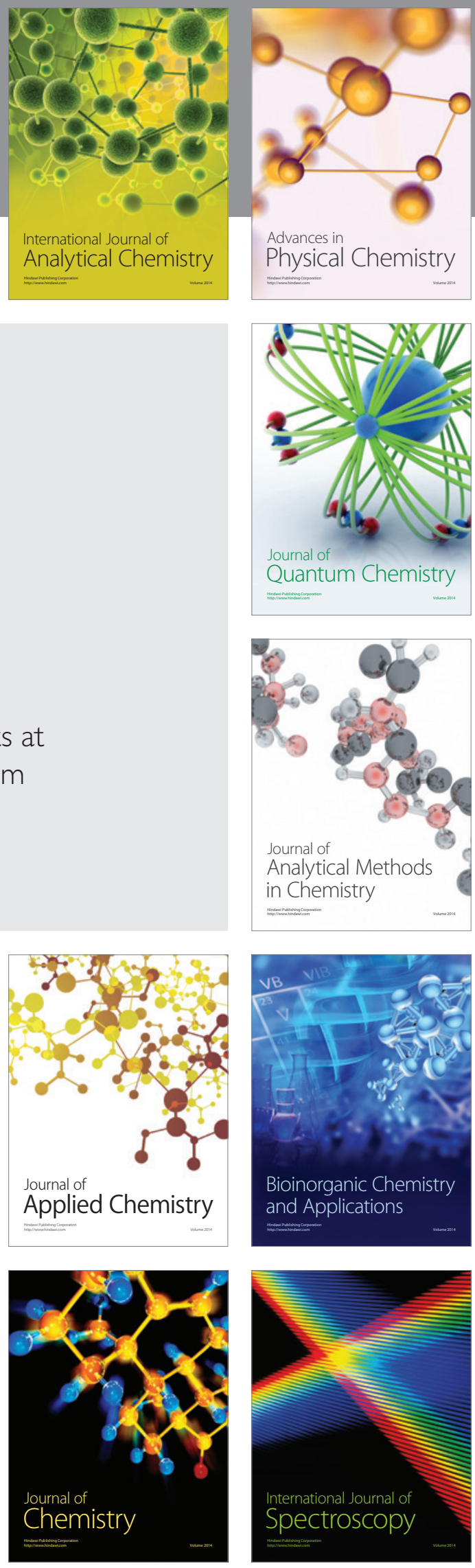